\title{
Effect of Deposition Condition on Composite Supercapacitor of Hydrous Ruthenium Oxides and Carbon-Nanotube Coatings by Cathodic Deposition Method
}

\author{
Yuli Lin and H.-S Hwang
}

The Institute of Engineering Science

Chung Hua University, Hsinchu, 300 Taiwan ROC

Supercapacitors have many advantages using in electrical devices for their larger capacitance - high power density and long cycle life. In general, supercapacitors can be classified into two categories, namely, pseudo-capacitors and double-layer capacitors. The fomer stores electrical charges in electrode surface region by faradaic reaction. While, in the latter, electrical charges are stored ate the double-layer formed at electrode/electrolyte interface. Various methods have been utilized to manufacture electrode including cyclic voltammetric method 、 sol-gel method 、 anodizing 、 cathodic deposition method and etc.[1].

In this study, hydrous ruthenium oxide with carbon nanotube additives was deposited on Ti substrate by cathodic deposition method. Ti substrate was first cleaned thoroughly by acetone and followed by chemical etching of $5 \% \mathrm{HF}$ for 5 minutes and $50 \% \mathrm{HCl}$ for 15 minutes. The purpose of adding carbon nanotube is to increase the effective area of hydrous ruthenium oxide coatings. The concentration of carbon nanotube added in the deposition process is $0.25 \mathrm{wt} \%$. The time of specimens which were immersed into the deposition bath varied from 10 minutes to 60 minutes. The electrical capacity characteristics of coating were examined by cyclic voltammetry. The microstructure of hydrous ruthenium oxide coating was also observed by scanning electron microscopy.

Figure 1(a)-(d) show the microstructure of hydrous ruthenium oxide coating with various deposition times. The capacitance of specimen with 10 minutes of deposition period was measured about 590 $\mathrm{F} / \mathrm{g}$ which is the maximum value in this study. It should be noted that the weight measurement contains both the hydrous ruthenium oxide and the carbon-nanotube. The measured capacitance will be $655.5 \mathrm{~g} / \mathrm{F}$ if the weight of carbon nanotube is deducted. Figure 2 reveals the difference of electrical capacity characteristics of specimens with various deposition times. It should be also noted that the larger area in $\mathrm{CV}$ curve was not corresponded to high capacitance. It means the weight of coating is very important. Figure 3 reveals the distribution of capacitance as increasing the deposition period. It was found that the measured capacitance was decreased when the deposition 
period was increased. The reason could be that the longer the deposition time, more weight of hydrous oxide coating can be deposited onto the surface of carbon nanotube. As a result, the measured capacitance decreases when the deposition time was increased.

[1] H.-S Hwang, Master Thesis, Chung-Hua University, 2004.

[2] Yuli Lin and H.-S Hwang, Microscopy and Microanalysis, Vol. 11(suppl.2), p1756-1757(2005).

[3] This research was supported by NSC94-2216-E-216-009, CHU94-2216-E-216-009 and CHU94-TR-09.
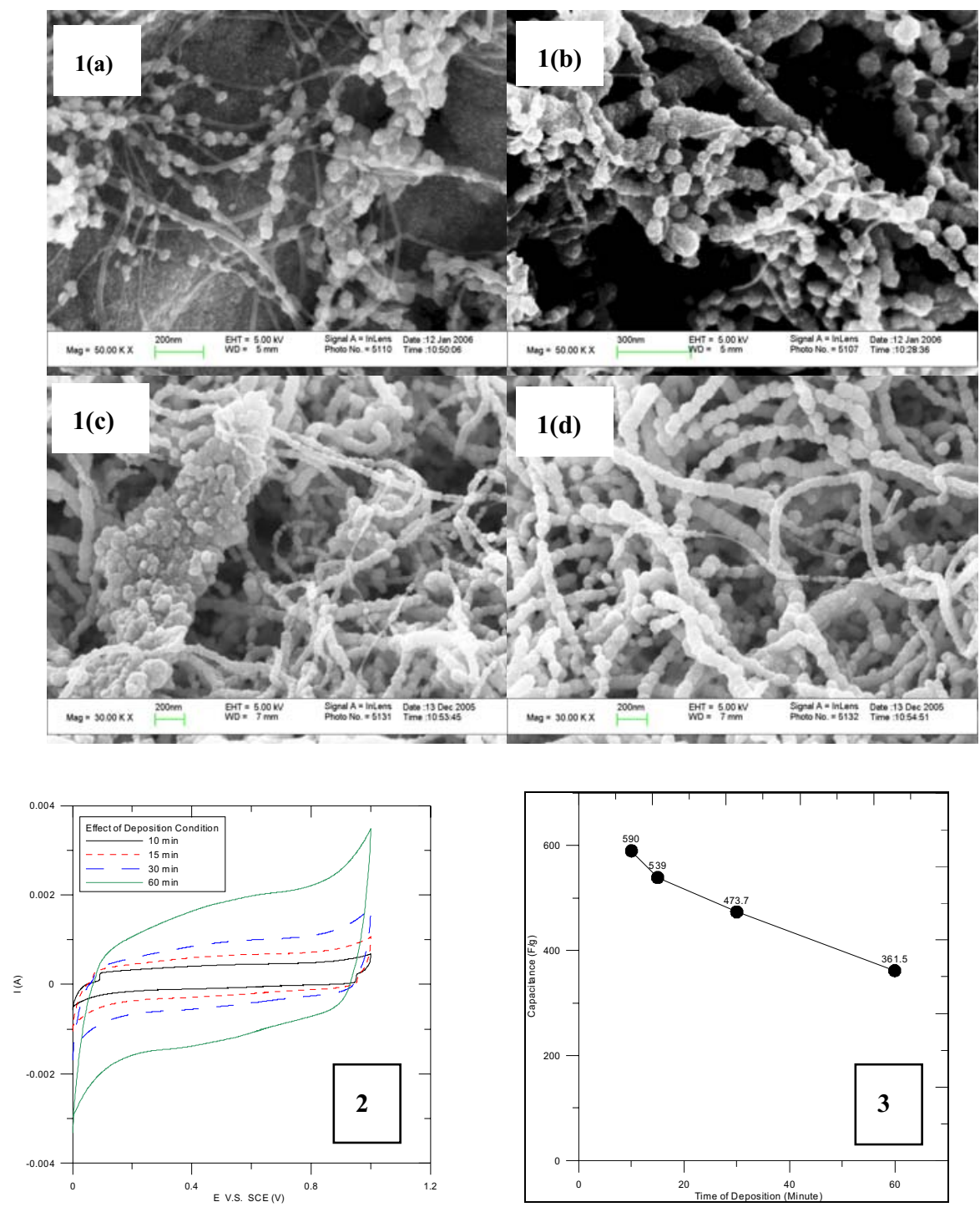

Figure 1 (a)-(d) The microstructure of specimen with various deposition times; 10 minutes, 15 minutes, 30 minutes and 60 minutes, respectively.

Figure 2 The electrical capacity characteristics of various deposition times

Figure 3 The distribution of capacitance with various deposition times. 